\title{
Irradiator Commissioning and Dosimetry for Assessment of LQ $\alpha$ and $\beta$ Parameters, Radiation Dosing Schema, and in vivo Dose Deposition
}

\author{
Samuel A. Sprowls ${ }^{*}, 1$, Vincenzo J. Pizzuti ${ }^{*}, 1,3$, William Pentz ${ }^{1}$, Divine C. Nwafor ${ }^{3,4}$, R. Alfredo C. Siochi ${ }^{2,3}$, Paul R. Lockman ${ }^{1}$ \\ ${ }^{1}$ Department of Basic Pharmaceutical Sciences, School of Pharmacy, West Virginia University ${ }^{2}$ Radiation Oncology, West Virginia University ${ }^{3}$ School of \\ Medicine, West Virginia University ${ }^{4}$ Department of Neuroscience, West Virginia University \\ *These authors contributed equally
}

Corresponding Author

Paul R. Lockman

prlockman@hsc.wvu.edu

\section{Citation}

Sprowls, S.A., Pizzuti, V.J., Pentz, W., Nwafor, D.C., Siochi, R.A.C.,

Lockman, P.R. Irradiator Commissioning and Dosimetry for Assessment of LQ $\alpha$ and $\beta$ Parameters, Radiation Dosing Schema, and in vivo Dose Deposition. J. Vis. Exp. (169), e61692, doi:10.3791/61692 (2021).

\section{Date Published}

March 11, 2021

DOI

$10.3791 / 61692$

URL

jove.com/video/61692

\section{Abstract}

Radiation dosimetry is critical in the accurate delivery and reproducibility of radiation schemes in preclinical models for high translational relevance. Prior to performing any in vitro or in vivo experiments, the specific dose output for the irradiator and individual experimental designs must be assessed. Using an ionization chamber, electrometer, and solid water setup, the dose output of wide fields at isocenter can be determined. Using a similar setup with radiochromic films in the place of the ionization chamber, dose rates for smaller fields at different depths can also be determined. In vitro clonogenic survival assays of cancer cells in response to radiation treatment are inexpensive experiments that provide a measure of inherent radio-sensitivity of cell lines by fitting these data with the traditional linear-quadratic model. Model parameters estimated from these assays, combined with the principles of biologic effective doses, allows one to develop varying fractionation schedules for radiation treatment that provide equivalent effective doses in tumor-bearing animal experiments. This is an important factor to consider and correct for in comparing in vivo radiation therapy schedules to eliminate potential confounding of results due to variance in the delivered effective doses. Taken together, this article provides a general method for dose output verification preclinical animal and cabinet irradiators, in vitro assessment of radiosensitivity, and verification of radiation delivery in small living organisms.

\section{Introduction}

Cancers collectively represent the second-leading cause of death in the U.S. and in many countries around the globe ${ }^{1}$. Radiation therapy is a cornerstone of treatment for many tumor subtypes and is administered to about half of all cancer patients $^{2,3}$. Patient outcomes for nearly all cancers have improved over time as equipment used to deliver radiation 
doses has steadily advanced and some effective multimodal therapy approaches were developed ${ }^{4,5,6}$, but recurrence and mortality rates for patients with certain types of tumors remain high ${ }^{7,8,9}$. Thus, radiotherapy for cancer continues to be an active area of basic and clinical research. Many pre-clinical radiotherapy studies employ the use of smallscale irradiators to deliver radiation doses to in vitro or animal models of cancers. With a multitude of potential experiments to conduct exploring mechanistic radiobiology details or novel treatments, common pitfalls may be encountered that lead to incorrect conclusions, poor reproducibility, and wasted resources. These pitfalls fall within three important areas: irradiator dosimetry, in vitro characterization of model cell lines, and in vivo irradiation dosing schedule and setup. Accurate and reproducible results from more advanced experiments are difficult to achieve without prior attention to these fundamental aspects of radiotherapy research.

The protocol detailed herein describes a generalized strategy for avoiding or mitigating these issues and draws upon several previously developed methodologies intended for independent use. These distinct methods have been merged so that a researcher interested in beginning or improving preclinical radiotherapy experiments could use this as a robust experimental layout. The suggested framework includes methodology for the commissioning of smallscale animal irradiators, for determining basic radiobiologic properties of model cancer cell lines, and for appropriately designing and administering a dosing and fractionation schedule for in vivo tumor models.

\section{Protocol}

Any steps of this protocol involving the use of laboratory animals, including handling and procedures, were approved by the Institutional Animal Care and Use Committee at West
Virginia University in Morgantown, West Virginia (Protocol number: 1604001894).

\section{Determination of dose output}

1. Use this protocol, based on the "In-phantom Method" protocol of the American Association of Physicists in Medicine Task Group (AAPM TG) $61^{10}$ and similar to the commissioning protocol set by Xstrahl, to determine beam output of the small animal irradiator with respect to particular geometry under the following setup conditions.

1. Set the irradiator to deliver radiation at $220 \mathrm{kVp}$ and $13 \mathrm{~mA}$, with an open field $(17 \mathrm{~cm}$ by $17 \mathrm{~cm})$ positioned at isocenter, or $35 \mathrm{~cm}$ from the source. Additionally, filter the beam with a $0.15 \mathrm{~mm}$ Cu filter with a broad focus. Some cellular irradiators contain only a radioactive source, this protocol can only be used for x-ray irradiators.

2. Align the solid water phantoms in the following order: $1 \mathrm{~cm}$ slab, $2 \mathrm{~cm}$ slab with ionization chamber slot, $2 \mathrm{~cm}$ slab, $1 \mathrm{~cm}$ slab. Stacking the solid water phantoms in this order positions the ionization chamber at a depth of $2 \mathrm{~cm}$, allowing $4 \mathrm{~cm}$ as well for backscatter. See Figure 1 for a graphical portrayal of the dosimetry setup.

NOTE: To accommodate the large, fairly heavy stack of solid water, the authors recommend the acquisition of a custom 3D printed couch with variable support to ensure the phantom stack is level and at the correct distance from the source across the surface of the material, not just at the center.

2. Use the measurement equipment (i.e., ADCL calibrated ionization chamber, electrometer) and an explanation 
of correction factors used can be found in Table of Materials and Table 1 respectively.

NOTE: That the ADCL provides values of $\mathrm{N}_{k}$ at a couple of points for different Half Value Layers (HVL, measure of beam quality). The value of $N_{k}$ to be used in the protocol should be based on an interpolation of the ADCL values for the unit's measured HVL. The manufacturer measured the HVL of our unit and we used that in our dose rate output determination.

3. Set up the phantom stack and insert the ionization chamber into the phantom as specified in step 1.1.2.

1. Adjust the phantom stack such that the source to surface distance (SSD), or the distance from the radiation source to the first surface, is $33 \mathrm{~cm}$ when appropriately levelled.

NOTE: The authors suggest creating a custom, 3D printed couch, large enough to support the dimensions of the solid water slabs. Additionally the one, utilized in this protocol has an adjustable component for levelling the phantom stack.

4. Take the average of three separate $x$-ray exposures, one minute readings with the electrometer bias voltage set at $300 \mathrm{~V}$. The result will be termed $\mathrm{M}^{+}$.

NOTE: Irradiations are performed with the instrument set to deliver radiation at $220 \mathrm{kVp}$ and $13 \mathrm{~mA}$. This is the same for the next two steps (steps 1.5-1.6). For user safety, ensure the doors remain closed during treatments.

5. Perform another set of three separate $\mathrm{x}$-ray exposures, 1 min readings with the electrometer bias voltage set at $-150 \mathrm{~V}$. The result will be termed $\mathrm{ML}_{\mathrm{L}}$.
6. Perform another set of three separate $x$-ray exposures, 1 min readings with the electrometer bias voltage set at $-300 \mathrm{~V}$. The result will be termed $\mathrm{MH}_{\mathrm{H}}$, or also $\mathrm{M}^{-}$.

7. Calculate $P_{p o l}$ and $P_{\text {ion }}$ using Equation 1 and Equation 2 respectively as described below:

$P_{\text {pol }}=\left(\left|M^{+}\right|+\left|M^{-}\right|\right) / 2\left|M^{-}\right|($Equation 1)

$P_{\text {ion }}=\left\{\frac{1-\left(\frac{V_{H}}{V_{L}}\right)^{2}}{\frac{M_{H}}{M_{L}}-\left(\frac{V_{H}}{V_{L}}\right)^{2}}\right\}=\left\{\frac{1-\left(\frac{-300}{-150}\right)^{2}}{\frac{M_{H}}{M_{L}}-\left(\frac{-300}{-150}\right)^{2}}\right\}=-3 /\left(M_{H} / M_{L}-4\right)$

(Equation 2)

8. Measure the temperature, in Celsius, and the pressure, in $\mathrm{kPa}$, inside the irradiator using a calibrated digital thermometer and barometer. Then, calculate PTP as indicated below in Equation 3.

NOTE: This calculation assumes that the ADCL used standard temperature and pressure values of $22{ }^{\circ} \mathrm{C}$ and $101.33 \mathrm{kPa}$ when stating their value for the air kerma calibration factor.

$P_{T P}=\left(\frac{273.2+T}{295.2}\right)\left(\frac{101.33}{P}\right)$ (Equation 3)

9. Calculate the corrected chamber reading, $M$, by multiplying the raw chamber reading, $\mathrm{M}_{\mathrm{H}}$, by $\mathrm{Pelec}_{\mathrm{e}}, \mathrm{P}_{\text {pol }}$, Pion, and PTP. This equation can be found below in Equation 4.

NOTE: This calculation assumes that the ADCL performed their calibration with the bias voltage set to $-300 \mathrm{~V}$, which is a fairly common practice.

$M=M_{H} P_{\text {elec }} P_{\text {pol }} P_{\text {ion }} P_{T P}$ (Equation 4).

10. Further multiply the corrected chamber reading by $\mathrm{N}_{\mathrm{k}}$, $\left[\left(\mu_{e n} / \mathrm{p}\right)^{\mathrm{w}} \text { air }\right]_{w a t e r}, \mathrm{PQ}_{\mathrm{Q}}$, cham, and $\mathrm{P}_{\text {sheath. }} \mathrm{P}$ sheath is only needed for measurements obtained in water. Therefore, for this protocol $P_{\text {sheath }}$ is just 1.

NOTE: Using the conditions in this protocol, the latter three items give a value of 1.0731 . This value depends on 
the beam quality, so the HVL must be known to determine it. The value of 1.0731 is specific to our unit and is given as an example. To determine the values of $P_{Q}$,cham and $\left[\left(\mu_{e n} / p\right)^{\mathrm{w}} \text { air }\right]_{w a t e r}$ specific to your unit, use the measured HVL and interpolate from Table VII, and Table VIII, and correct for the reference field size according to Figure 3 and Figure 4 from the AAPM TG61 protocol ${ }^{10}$. In our case, multiplying $N_{k}$ by 1.0731 provides the dose to water, $D_{W}$, in Gy for a nominal time of 1 min, assuming the ADCL $N_{k}$ value is given in Gy/Coulumbs.

11. Determine the end effect of the irradiator being used. When the x-rays are first generated, the output rises to its full value over some finite time. Similarly, when the $x$-ray source is turned off, the output decreases to zero over some finite time.

1. Account for the time for this transition, or the end effect. This can be done by taking the average of three readings with the electrometer bias voltage set at $-300 \mathrm{~V}$, for a variety of time settings. Do this for 6 , $12,18,24,30$, and 60 seconds.

2. Plot the electrometer readings against time and find the best straight line. The total time, $t$, for a 1 minute treatment can be calculated by equation 5 : $t=1+\delta t / 60$ (Equation 5).

12. Calculate the dose rate for a given irradiator by equation 6:

$\dot{D}=D_{w} /\left(1+\frac{6 t}{60}\right)$ (Equation 6)

\section{Creating a radiochromic film calibration curve}

1. For a list of necessary materials, see Table of Materials.

2. Using a near identical set up as the previous protocol, place the film at a $2 \mathrm{~cm}$ depth in the solid water phantom stack. The order of solid water phantoms is insignificant so long as there are $2 \mathrm{~cm}$ of solid water above and $4 \mathrm{~cm}$ of solid water below for buildup and backscatter effects.

3. Using the determined dose commissioned in protocol 1 , determine the treatment times for the doses listed in Table 2 using equation 7:

$T=\frac{D_{X}}{\dot{\mathrm{D}}} * \frac{60 s}{\min }$ (Equation 7)

4. Prepare several pieces of film ensuring that each film is of the same size and remains in the same orientation from treatment through scan acquisition. This can be done by placing a small diagonal cut in the lower left corner. Each film from this point forward must be from the same batch of film.

NOTE: Prepare 3 separate replicates for each dose point to be assessed.

5. Scan the cut pieces using a 48-bit color photo scanner with all corrections turned off. Ensure that each film is placed in the exact center of the scanning bed. The values obtained are the pre-exposure scans used for determining the unexposed optical density ${ }^{11,12}$. Save all images in the .Tiff file format to avoid compression of key data.

NOTE: The authors recommend scanning the films three times and using the obtained average as the single value for a given film.

6. Begin the irradiation of the films by placing a piece of film on top of $4 \mathrm{~cm}$ of solid water and positioned the remaining $2 \mathrm{~cm}$ of solid water above, as described previously in this section.

7. Adjust the phantom set so that the film is the same distance from the source as the ionization chamber was when determining the dose output. This is the isocentric point of the irradiator. 
8. Program the treatment time calculated in step 2.3 above for one prescribed dose.

9. Repeat treatment for each of the doses listed in Table 2.

10. Allow films to rest for 24 hours protected from light.

11. Acquire the post-exposure film scans in the same manner as above.

12. Import images to Image J analysis software and perform all measurements on the red channel.

1. Drag the image in .Tiff file format into ImageJ.

2. Click the Image drop down menu. Select Color from Image drop down menu. Select split channels from Color option.

3. Using only the red image channel, draw a region of interest using the rectangle tool. Press Ctrl+M. Transcribe mean value from the results window.

4. Repeat steps 2.12.1-2.12.4 for all scanned films.

13. Obtain the pixel value in a centrally located $1 \mathrm{~cm}$ by 1 $\mathrm{cm}$ square for both the unexposed and exposed films. These values will be denoted as $P V U(D)$ and $P V(D)$ respectively, and can be used to calculate the net optical density as described in Equation 8.

$O D_{n e t}=\log \left(\frac{P V_{U(D)}}{P V_{(D)}}\right)(\text { Equation 8) })^{13}$

14. Repeat step 2.13 for each pair of film images, both preexposure and post-exposure.

15. Plot a graph of the dose versus the net optical density and fit the curve to a cubic polynomial in the format of $y=a x^{3}$ $+b x^{2}+c x+d$. An example can be found in Figure 2B.

\section{Determination of $\alpha / \beta$ value for specific cancer cell lines via clonogenic assay}

NOTE: The following protocol is a modified version of the methods described by Franken et $\mathrm{al}^{14}$ and can be seen in Figure 3.

1. Grow cells to $\sim 80 \%$ confluency. Avoid using overconfluent sources of cells for this experiment, as it is necessary that the cells are in the log-phase of cell growth. For the representative clonogenic assay results displayed in Figure 3C, brain-tropic MDA-MB-231 breast cancer cells were cultured in Dulbecco's Modified Eagle Medium (DMEM) supplemented with $10 \%$ fetal bovine serum and penicillin/streptomycin and were incubated at $37^{\circ} \mathrm{C}$ and $5 \%$ carbon dioxide in a humidified incubator.

2. Seed the cells at the desired density for the colony assay. Accurate dilutions during the seeding are crucial to determining the assay's plating efficiency. Be sure to plate multiple replicates.

3. Proceed with this step if radiation treatment will precede cell plating (Figure 3A). Alternatively, proceed to step 3.4 if cell plating will precede radiation treatment.

1. Perform desired radiation treatment on culture flasks. Any additional treatments (i.e. drug treatments) may be performed at any point before or after this. For the representative results in Figure 3C, radiation treatment occurred after plating cells, detailed in step 3.4 .

2. Extract the cells using preferred trypsinization method and create a single cell suspension. Remove culture media and add recombinant enzyme (e.g., TrypLE Express) to detach cells from the flask. Incubate cells with the enzyme for 
approximately 3 minutes until cells were detached as detected using a light microscope. Neutralize the enzyme using an equal volume of cell culture media. Centrifuge cells at $300 \times \mathrm{g}$ for $10 \mathrm{~min}$ and resuspend to the desired concentration in culture medium.

3. Plate the cells at desired densities in multiple replicates.

4. Replace with fresh media after the first $24 \mathrm{~h}$.

5. Continue to replace media every 2-3 days.

6. Continue culturing cells until control colonies exceed 50 cells per colony, $\sim 9-14$ days. Control colonies are those treatment groups that receive no radiation doses. For experiments using drug treatments as well, another control group with drug dosing but no radiation treatment will also be required.

4. Proceed with this step when seeding cells before radiation treatment (Figure $3 \mathrm{~B}$ ).

1. Extract cells using preferred trypsinization method and create a single cell suspension.

2. Place cells at desired densities in multiple replicates.

3. Allow cells to adhere to plate overnight.

4. Perform desired radiation doses. Additional treatments, such as drug dosing, may be performed at any point before or after this step, so long as cells have attached to their treatment plates. For the representative results in Figure 3C, 1250 brain-tropic MDA-MB-231 cells were plated prior to treatment (step 3.4). Then, cells were treated with $15 \mathrm{nM}$ doxorubicin 3 hours prior to irradiation with 3 Gy of X-rays.

5. Replace the media after the initial $24 \mathrm{~h}$.

6. Replace media every 2-3 days.
7. Culture the treated cells until control group colonies exceed 50 cells, $\sim 9-14$ days. Control colonies are those treatment groups that receive no radiation doses. For experiments using drug treatments as well, another control group with drug dosing but no radiation treatment will also be required.

5. Remove culture media from wells or dishes, and wash with PBS.

6. Fix cells for 15 minutes in a 1:7 (v:v) solution of glacial acetic acid and methanol.

7. Remove the fixation solution.

8. After fixation, stain cells for 30 minutes, or $2 \mathrm{~h}$ if time is available, at room temperature with a $2.5-5.0 \mathrm{mg} / \mathrm{mL}$ solution of crystal violet in a 4:1 (v:v) solution of distilled water and methanol.

9. Remove staining solution and wash cells in a large, roomtemperature water bath.

NOTE: Do not wash under running water.

10. Count the resultant number of colonies in each treatment group and calculate the survival fraction of each plate.

11. Plot the survival fraction against the corresponding dose delivered, and fit the curve with an exponential fit.

12. To estimate the $\alpha / \beta$ value, use an exponential fit of the above plot to estimate the values for each of the adjustable parameters in the linear-quadratic equation found below:

$S F=e^{-\alpha * D-\beta * D^{2}}$ (Equation 9)

NOTE: Irradiation of cells can typically be done at isocenter without any collimation provided the field size is large enough to accommodate well-plates or petri dishes. Potential pitfalls in this protocol may include yields such as no colony formation, significant cell migration with 
clear cell growth but no true colonies, or contamination due to treatment in a non-sterile irradiator chamber.

\section{Determination of the specific dose output for variable experimental designs}

1. Decide upon the desired field size and distance from source.

NOTE: Collimation will alter the dose rate no matter the size or distance of the collimator from the $\mathrm{x}$-ray source.

2. Using solid water phantoms to provide buildup and backscatter, position a piece of film in the correct orientation that best portrays the experimental design.

NOTE: For any experimental setup solid water may not provide the most accurate representation of a given design. Instead we recommend using the actual experiment's vessels (i.e., Petri dish, well-plates, small animal phantoms, etc.).

3. Irradiate films for $1(\mathrm{~N}=3)$ and $2(\mathrm{~N}=3)$ minutes.

4. Allow films to rest for 24 hours protected from light.

5. Determine the net optical density of each the films following the procedures from Section 2. Use the film calibration curve to determine dose from the net optical density.

6. Determine the dose at 1 minute, $D_{1}$, as the output dose rate, $\dot{D}$, for this experimental setup defined by Equation 10 as follows:

$D_{\exp 1}=D_{1} / 1 \min ($ Equation 10)

7. Similarly, caluclate the dose at 2 minutes by Equation 11 as follows:

$D_{\exp 2}=D_{2} / 2 \min ($ Equation 11)

8. Due to the end effect, the dose rate for the above calculations may be slightly different. For this reason to calculate $D_{\exp }$ for the desired experimental design, use an average of the individual $D_{\exp }$ as indicated in Equation 12:

$D_{\text {exp }}^{\text {avg }}=\left({ }^{D_{\exp } / 1 \mathrm{~min}}+{ }^{D_{\exp 2} / 2 \mathrm{~min}}\right) / 2$ (Equation 12)

9. Using this average, define the time for treating to any desired dose for this particular setup in Equation 13:

$T=D_{\text {exp }}^{\text {avg }} / D$ (Equation 13)

\section{Treating mice bearing tumors in anatomical location of interest}

1. Anesthetize mouse with safe and humane anesthesia techniques approved by the institution's IACUC.

2. Place anesthetized animal in restraint as indicated in the desired experimental design.

3. This step is optional, if not available proceed to step 5.6. Obtain a radiogram, using an onboard portal camera, of the mouse without collimation using an aluminum filter.

4. Obtain a second radiogram with collimation in place.

5. Overlay radiograms in ImageJ to demonstrate beam positioning.

6. Using the predetermined $\alpha / \beta$ value, determine the dose scheme that provides the most reasonable approach to answer a research question (i.e., if wanting to model the effects of a dose of $30 \mathrm{~Gy}$ delivered in 10 fractions of 3 Gy, but only wish to give four fractions). Using equation 14 , with an assumed $\alpha / \beta$ value of 10 (this value can be determined for individual cancer cell lines in protocol 3) and a BED similar to that of $30 \mathrm{~Gy} / 10 \mathrm{~F}$, treat with $24 \mathrm{~Gy}$ in 4 fractions of 6 Gy.

7. Treat animal for the prescribed time given for the desired dose. 


\section{Histological confirmation of dose deposition in vivo}

1. Following protocol 5, collect tissue of interest within 1 hour of treatment ${ }^{15,16}$. Following tissue harvest, proceed with preferred immunohistochemistry protocol. An example is given below.

2. Perfuse animal with ice cold $4 \%$ paraformaldehyde (PFA).

3. Post-fix in PFA at $4{ }^{\circ} \mathrm{C}$.

4. Following fixation, fix tissue sequentially in $10 \%, 20 \%$, and $30 \%$ sucrose for 24 hours each at room temperature.

5. Embed tissue in gelatin and sequentially fix in 4\% PFA and again in $10-30 \%$ sucrose for 24 hours each at room temperature.

6. Trim block and place at $-80^{\circ} \mathrm{C}$ for 30 minutes.

7. Slice tissue into $20-30 \mu \mathrm{m}$ sections.

8. Immunostain slides as free floating sections in a six wellplate $^{17,18}$.

1. Wash three times and permeabilize for 30 minutes on a shaker with $1.83 \%$ lysine in $1 \%$ Triton, and $4 \%$ heat-inactivated goat serum.

2. Incubate sections with anti-yH2AX antibody for 24 hours, followed by a 2 hour incubation with desired secondary antibody.

3. Coverslip slides with glass coverslips using preferred mounting media.

9. Image on a fluorescent microscope.

\section{Representative Results}

Following protocol 1 will provide a dose rate in $\mathrm{Gy} / \mathrm{min}$, which is specific to the irradiator being used. However, regardless of the type of irradiator, with a known dose rate a calibration curve can be generated using protocol 2 yielding similar films and a similar calibration curve to that in Figure 2A-B. A successful assay from protocol 3 will yield distinct, welldemarcated colonies of cells that stain homogenously violet. The estimate of $\alpha / \beta$ can be compared to literature values or other treatment groups to interpret the radio-sensitivity of the given cell line. Utilizing the calibration curve developed following protocol 2 and displayed in Figure 2B, protocol 4 will yield two film samples resembling Figure 2A that can be used to estimate required experimental irradiation times. If an on-board portal imaging camera is available for the irradiator being used, radiograms of small animals can be obtained with and without collimation. Overlaying these images will demonstrate the exact positioning of the collimated radiation beam relative to the small animal being treated as depicted in Figure 4A. Successful dose-deposition in protocol 5 can be confirmed following protocol 6 . One indication that radiation is being deposited in an in vivo or in vitro systems is through the detection of double stranded DNA breaks. Illustrated in Figure 4B, the same mouse treated solely through the right hemisphere in Figure 4A, demonstrates positive $\mathrm{yH} 2 \mathrm{AX}$ staining only in the treated hemisphere. In this figure, the nuclei are stained with DAPI to show two things; 1) the whole are of the brain which the anti $\mathrm{yH} 2 \mathrm{AX}$ antibody was applied to during histological analysis, and 2) the untreated hemisphere of the brain remains unstained. 


\section{Inside}

\section{Cabinet}

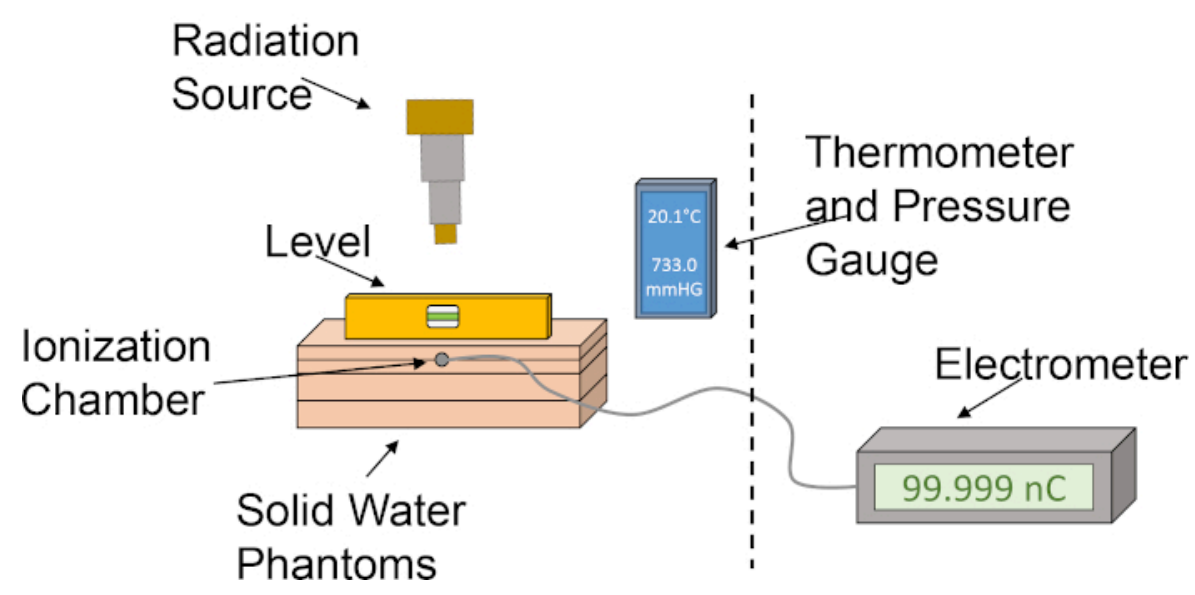

Figure 1: Rough set up of ionization chamber and water phantom set up for determination of dose output. The pictogram illustrates a basic setup utilizing the various components required for dosimetry using an ionization chamber and solid water phantoms within the cabinet of the irradiator. Please click here to view a larger version of this figure.
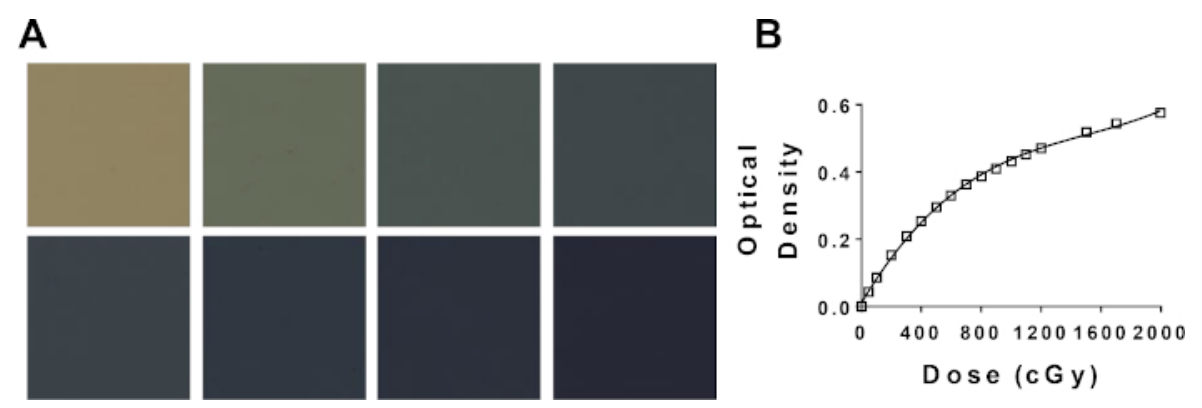

Figure 2: Generation of a calibration curve using radiochromic film. (A) Representative color change of radiochromic film with increasing dose. Top left (0 cGy); bottom right (2000 cGy). (B) Potential radiochromic film calibration curve comparing net optical density and dose. Please click here to view a larger version of this figure. 

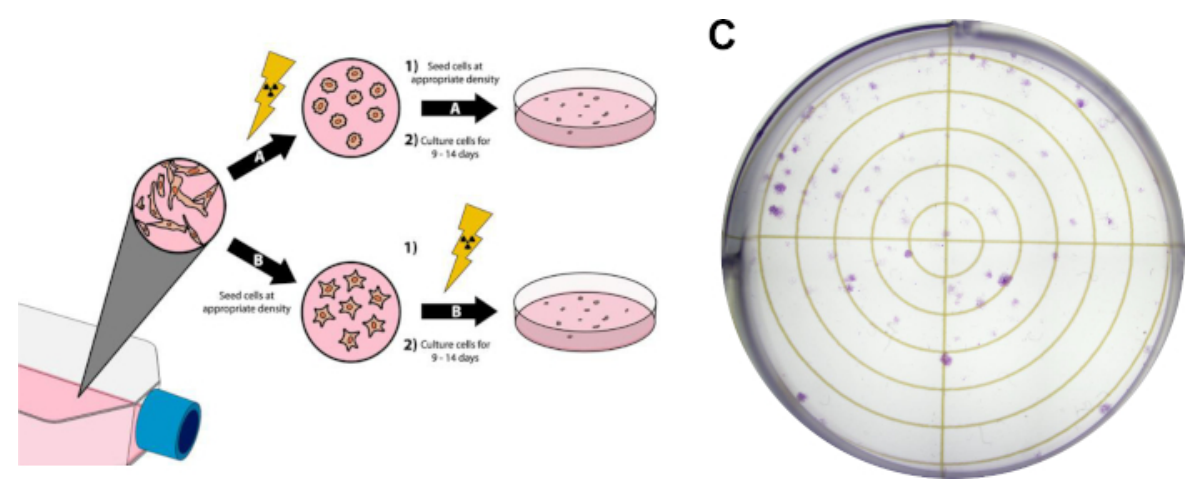

Figure 3: Clonogenic Assay of Cancer Cells. Radiation treatment of cells can be done prior to plating in six well plates/ petri dishes $(\mathbf{A})$, or after (B). In panel (C), a representative image is displayed of a successful clonogenic assay with MDAMB-231 breast cancer cells after following Protocol Section 3. Please click here to view a larger version of this figure.
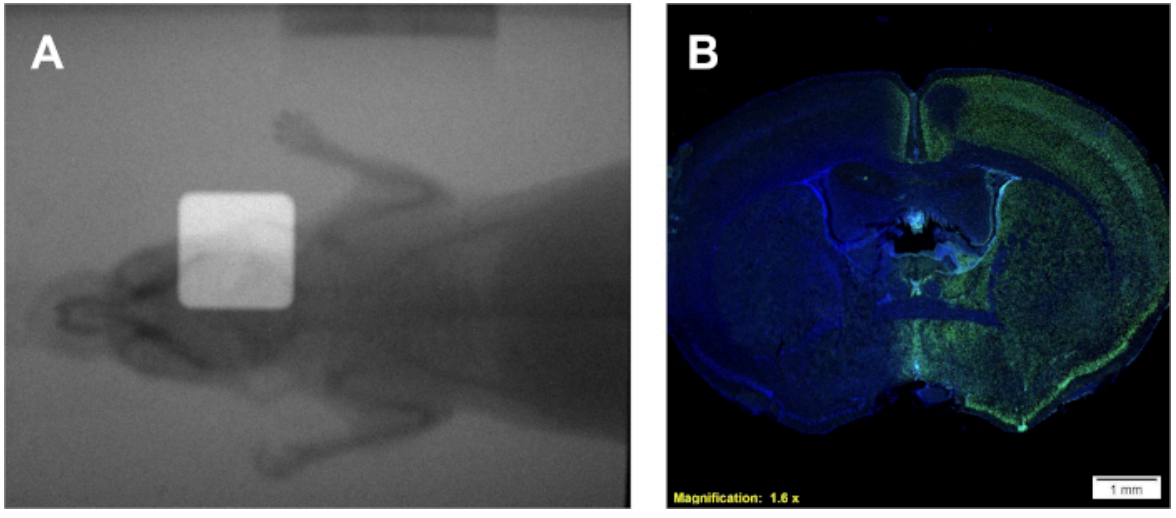

Figure 4: Use of dual overlayed radiograms for positioning (if available) and positive $\mathrm{YH} 2 \mathrm{AX}$ immunohistochemical staining for confirmation of dose deposition. (A) Representative overlayed radiograms depicting placement of radiation beam. (B) Representative results indicating dose deposition to the right hemisphere as demonstrated by increased $\mathrm{yH} 2 \mathrm{AX}$ intensity. Please click here to view a larger version of this figure. 


\begin{tabular}{|c|c|}
\hline Correction Factor & Explanation \\
\hline Nk & Air kerma calibration factor \\
\hline$\left[\left(\mu_{e n} / \rho\right)^{W}\right.$ air $]$ water & Ration of mass energy absorption coefficients of water to air; approximately 1.05 \\
\hline$P_{q}$, Cham & $\begin{array}{l}\text { Correction accounting for chamber stem affecting photon } \\
\text { fluence perterbation by chamber; approximately } 1.022\end{array}$ \\
\hline Psheath & $\begin{array}{l}\text { Correction accounting for sheath protecting ionization } \\
\text { chamber; value of } 1 \text {, as chamber is waterproof }\end{array}$ \\
\hline Ppol & Correction factor accounting for polarity; determined in Protocol 1 \\
\hline Pion & Correction factor accounting for ion recombination; determined in Protocol 1 \\
\hline PTp & $\begin{array}{c}\text { Correction factor acocunting for temerpature and } \\
\text { pressure on day of experiment; determined in Protocol } 1\end{array}$ \\
\hline
\end{tabular}

Table 1: Correction factors needed for determination of dose rate in Protocol 1.

\begin{tabular}{|c|c|}
\hline Dose & N \\
\hline 0.5 & 3 \\
\hline 1 & 3 \\
\hline 2 & 3 \\
\hline 3 & 3 \\
\hline 4 & 3 \\
\hline 6 & 3 \\
\hline 8 & 3 \\
\hline 10 & 3 \\
\hline $12^{*}$ & 3 \\
\hline $15^{*}$ & 3 \\
\hline $20^{*}$ & \\
\hline$*$ Only necessary for doses exceeding 10 for individual experiments. \\
\hline
\end{tabular}

Table 2: Doses to be used in generation of radiochromic film calibration curve. 


\section{Discussion}

The above protocol describes a user-friendly approach for radiation dosimetry, determination of $\alpha / \beta$ values in cancer cell lines, and a brief example of an approach for irradiation in a preclinical model of breast cancer brain metastasis. These methods can be used to study any model of cancer and are not just limited to brain metastasis of breast cancer. In this section we will discuss the relevant intricacies underlying preclinical radiotherapy experiments.

Dosimetry involves two parts: 1) calibrate the output with a farmer chamber, so that the dose rate of the $x$-ray unit is established, and 2) prepare a practical dosimetry measurement system using radiochromic film. With regards to output calibration, TG-61 provides a reproducible method in water. The protocol here uses Gammex RMI 457 solid water, as recommended by XStrahl, the manufacturer of the irradiator. Although relative dosimetry (profiles or depth dose curves normalized to maximum dose) analysis with solid water, agrees to better than $1 \%$ with that of water, there is a difference of about 3 to $4 \%$ in absolute dose due to a higher mass energy absorption coefficient for solid water compared to water. However, as all installations of the XStrahl system use the solid water protocol for output calibration, we did not correct for these differences. Knowing the output allows the calculation of the exposure time required to deliver a desired dose. Placing film in the same setup as the farmer chamber allows us to deliver known doses to the film. Scanning the film then provides optical densities. The dose to the film can then be graphed against the corresponding net optical density (difference in optical density after and before exposure). This produces a film calibration curve. When we change experimental setups, the dose rate in that situation could change, since dose rate depends on field size, depth and the material being irradiated. Exposing film with the experimental setup provides us with a net optical density, and using the film calibration curve, we can then determine the corresponding dose. Dividing this dose by the time the film was irradiated, we get the dose rate. This dose rate can then be used to calculate the exposure time to deliver a desired dose for the given experimental setup. The protocol described above handles several nuances associated with film dosimetry. For example, after exposure, the film requires approximately 24 hours for the chemical reactions in the film's active layer to be virtually complete. Not waiting for this amount of time will lead to a lower optical density.

For any study to have reproducible dosimetry it is important to know and understand several of the key elements of a given irradiator. In particular, it is crucial to know and detail to other researchers the make and model of the irradiator used, the source type (x-ray, radioactive, etc.), energy, half-value layer, field size, source to surface and source to isocenter distances, size of material irradiated, attenuation before and backscatter after the irradiated material, experiment-specific dose rate, fractionation schema, exact dosimetry equipment utilized, and the dosimetry protocol used. All of these points of information are what cohesively describe the beam quality of a given irradiator prior to delivering a dose to any animal or cell $^{19}$. Another pertinent point of information from this protocol and others is that the dose rate achieved in Protocol $\mathbf{1}$ is simply the output of the irradiator being used. For any given experiment it is important to define the dose rate for that particular setup (Protocol 4) by comparison with a generated radiochromic film calibration curve (Protocol 2).

In vitro experimentation provides important details about the radiobiologic behavior of cancer cell lines. In vitro clonogenic cell survival assays accurately estimate and quantify the inherent radio-sensitivity of a cell line ${ }^{20}$, aiding in 
the design of fractionation schedules in subsequent cellular or small animal experiments ${ }^{21}$. Specifically, these assays approximate values for the parameters $\alpha$ and $\beta$ that are used in the linear-quadratic model to predict cell death in response to radiotherapy according to the equation:

$S F=e^{-\alpha * D-\beta * D^{2}}$ (Equation 9)

where SF is the surviving fraction of clonogenically viable cells, $D$ is radiation dose in $G y$, and $\alpha$ and $\beta$ are fitted parameters $^{22}$. The ratio $\alpha / \beta$ provides an inherent measure of cellular radio-sensitivity, with higher values correlating with increased sensitivity of a cell line ${ }^{22}$. Because this functional relationship is non-linear with respect to dose, the biologic effects of a radiotherapy fractionation scheme are not only related to the total delivered dose but also the number and size of fractions ${ }^{23}$. The biologic effective dose (BED) is a measure of the true biological dose delivered to a tissue and permits direct comparison of different fractionations schemes $^{24,25}$. The BED equation only requires an estimate of $\alpha / \beta$, and is displayed below:

$B E D=n * D *\left[1+\frac{D}{\alpha / \beta}\right]($ Equation 14)

where $\mathrm{n}$ is the number of fractions of dose D. Clonogenic cell survival assays estimate $\alpha / \beta$ and facilitate the direct comparison of radiotherapy fractionation schemes via the BED equation. Incorrect conclusions may be drawn regarding a tissue or organ response to radiotherapy (or combinations of radiotherapy with other modalities) if the BED in the treatment groups is not equitable within or between experiments. For example, 2 fractions of 10 Gy compared with 4 fractions of 5 Gy do not yield the same BED, and thus these dosing schemes cannot be directly compared in terms of biologic response. The BED equation, while imperfect due to inherent limitations in the linear-quadratic model, reliably estimates equitable effects for a wide range of experimental treatment conditions $^{24,25}$.

Clonogenic cell survival assays clearly play an important role in studying radiotherapy effects in cancer models, but in vitro experimentation offers a number of additional options to further explore mechanistic details of cancer cell radiobiology. Simple modifications of the clonogenic cell survival assay were used to determine the modes of action for some radio-sensitizing chemotherapies, such as paclitaxel or etoposide 26,27 . Further in vitro experimental options include immunocytochemistry studies to examine specific cellular repair pathways, such as $\mathrm{y}-\mathrm{H} 2 \mathrm{AX}$ foci and/or 53BP1 staining for double-stranded DNA break repair ${ }^{28}$. These experiments may be of particular interest when comparing radiotherapy as a single modality with combination therapies, especially when probing mechanistic details for a given cell line. Other experimental options include cytokine measurements to examine the innate role of a cell's inflammatory response to irradiation or analyses of the mode of cell death (i.e., apoptosis, necrosis, mitotic catastrophe, etc.) under different therapeutic conditions $29,30,31$. This type of experimentation can complement or replace animal experimentation and provide a more complete understanding of a cancer cell line's radiobiology. Regardless of the choice of additional experiments to conduct, a standard clonogenic cell survival assay as described in protocol 3 is an important initial radiobiologic assessment of a cell line.

Clonogenic assays and radiation dosimetry provide the researcher with a means to precisely plan experiments to more directly resemble clinical scenarios. With the addition of preclinical cancer small rodent models, it is possible to study the response to radiation alone or in the context of a treatment plan in vivo. Prior to using animals, it is important to determine 
the relative dose output of the specific setup if it differs from the setup used for determination of dose output ${ }^{32,33}$. When it comes to determining a dose rate for field sizes of $<10 \mathrm{~mm}$, use of an ionization chamber becomes less accurate due to alignment within a small field and partial volume averaging effects $^{33}$. The use of radiochromic film to determine output in combination with in vivo immunohistochemical experiments has been used to determine output and dose deposition in the past $^{16,34,35,36,37,38}$.

\section{Disclosures}

The authors have no disclosures to make.

\section{Acknowledgments}

The authors would like to thank the Microscope and Animal Models Imaging Facilites at WVU for the use of their equipment supported by grant number P20GM103434. Additionally, this work was supported by grant number P20GM121322 from the National Institue of General Medical Sciences, by National Cancer Institute grant number F99CA25376801, and the Mylan Chair Endowment Fund.

\section{References}

1. Siegel, R. L., Miller, K. D., Jemal, A. Cancer statistics, 2019. CA: A Cancer Journal for Clinicians. 69 (1), 7-34 (2019).

2. Allen, C., Her, S., Jaffray, D. A. Radiotherapy for Cancer: Present and Future. Advanced Drug Delivery Reviews. 109 1-2 (2017).

3. Delaney, G., Jacob, S., Featherstone, C., Barton, M. The role of radiotherapy in cancer treatment: estimating optimal utilization from a review of evidence-based clinical guidelines. Cancer. 104 (6), 1129-1137 (2005).
4. Chen, H. H. W., Kuo, M. T. Improving radiotherapy in cancer treatment: Promises and challenges. Oncotarget. 8 (37), 62742-62758 (2017).

5. Le, Q. T., Shirato, H., Giaccia, A. J., Koong, A. C. Emerging Treatment Paradigms in Radiation Oncology. Clinical Cancer Research. 21 (15), 3393-3401 (2015).

6. Baumann, M. et al. Radiation oncology in the era of precision medicine. Nature Reviews Cancer. 16 (4), 234-249 (2016).

7. Leeman, J. E. et al. Patterns of Treatment Failure and Postrecurrence Outcomes Among Patients With Locally Advanced Head and Neck Squamous Cell Carcinoma After Chemoradiotherapy Using Modern Radiation Techniques. JAMA Oncology. 3 (11), 1487-1494 (2017).

8. Coy, P. et al. Patterns of failure following loco-regional radiotherapy in the treatment of limited stage small cell lung cancer. International Journal of Radiation Oncology - Biology • Physics. 28 (2), 355-362 (1994).

9. Achrol, A. S. et al. Brain metastases. Nature Reviews Disease Primers. 5 (1), 5 (2019).

10. Ma, C. M. et al. AAPM protocol for $40-300 \mathrm{kV} x-$ ray beam dosimetry in radiotherapy and radiobiology. Medical Physics. 28 (6), 868-893 (2001).

11. Wang, Y. F., Lin, S. C., Na, Y. H., Black, P. J., Wuu, C. S. Dosimetric verification and commissioning for a small animal image-guided irradiator. Physics in Medicine and Biology. 63 (14), 145001 (2018).

12. Wack, L. et al. High throughput film dosimetry in homogeneous and heterogeneous media for a small animal irradiator. Physical Medicine. 30 (1), 36-46 (2014). 
13. Devic, S. Radiochromic film dosimetry: past, present, and future. Physical Medicine. 27 (3), 122-134 (2011).

14. Franken, N. A., Rodermond, H. M., Stap, J., Haveman, J., van Bree, C. Clonogenic assay of cells in vitro. Nature Protocols. 1 (5), 2315-2319 (2006).

15. Ford, E. C. et al. Localized CT-guided irradiation inhibits neurogenesis in specific regions of the adult mouse brain. Radiation Research. 175 (6), 774-783 (2011).

16. Zarghami, N. et al. Half brain irradiation in a murine model of breast cancer brain metastasis: magnetic resonance imaging and histological assessments of dose-response. Radiation Oncology. 13 (1), 104 (2018).

17. Nwafor, D. C. et al. Loss of tissue-nonspecific alkaline phosphatase (TNAP) enzyme activity in cerebral microvessels is coupled to persistent neuroinflammation and behavioral deficits in late sepsis. Brain, Behavior, and Immunity. (2019).

18. Amtul, Z., Hepburn, J. D. Protein markers of cerebrovascular disruption of neurovascular unit: immunohistochemical and imaging approaches. Reviews in Neuroscience. 25 (4), 481-507 (2014).

19. Draeger, E. et al. A Dose of Reality: How 20 Years of Incomplete Physics and Dosimetry Reporting in Radiobiology Studies May Have Contributed to the Reproducibility Crisis. International Journal of Radiation Oncology • Biology • Physics. 106 (2), 243-252 (2020).

20. Dunne, A. L. et al. Relationship between clonogenic radiosensitivity, radiation-induced apoptosis and DNA damage/repair in human colon cancer cells. British Journal of Cancer. 89 (12), 2277-2283 (2003).
21. Yang, Y., Xing, L. Optimization of radiotherapy dose-time fractionation with consideration of tumor specific biology. Medical Physics. 32 (12), 3666-3677 (2005).

22. Hall, E. J., Giaccia, A. J. Radiobiology for the radiologist. Eighth edition. edn Wolters Kluwer, (2019).

23. van Leeuwen, C. M. et al. The alfa and beta of tumours: a review of parameters of the linear-quadratic model, derived from clinical radiotherapy studies. Radiation Oncology. 13 (1), 96 (2018).

24. Fowler, J. F. 21 years of biologically effective dose. British Institute of Radiology. 83 (991), 554-568 (2010).

25. Jones, B., Dale, R. G., Deehan, C., Hopkins, K. I., Morgan, D. A. The role of biologically effective dose (BED) in clinical oncology. Clinical Oncology journal| The Royal College of Radiologists. 13 (2), 71-81 (2001).

26. Choy, H., Rodriguez, F. F., Koester, S., Hilsenbeck, S., Von Hoff, D. D. Investigation of taxol as a potential radiation sensitizer. Cancer. 71 (11), 3774-3778 (1993).

27. Ng, C. E., Bussey, A. M., Raaphorst, G. P. Inhibition of potentially lethal and sublethal damage repair by camptothecin and etoposide in human melanoma cell lines. International Journal of Radiation Biology. 66 (1), 49-57 (1994).

28. Kurashige, T., Shimamura, M., Nagayama, Y. Differences in quantification of DNA double-strand breaks assessed by 53BP1/gammaH2AX focus formation assays and the comet assay in mammalian cells treated with irradiation and $\mathrm{N}$-acetyl-L-cysteine. Journal of Radiation Research. 57 (3), 312-317 (2016).

29. Schaue, D., Kachikwu, E. L., McBride, W. H. Cytokines in radiobiological responses: a review. Radiation Research. 178 (6), 505-523 (2012). 
30. Mery, B. et al. In Vitro Cell Death Determination for Drug Discovery: A Landscape Review of Real Issues. Journal of Cell Death. 101179670717691251 (2017).

31. Galluzzi, L. et al. Molecular mechanisms of cell death: recommendations of the Nomenclature Committee on Cell Death 2018. Cell Death \& Differentiation. 25 (3), 486-541 (2018).

32. Felix, M. C. et al. Collimator optimization for small animal radiation therapy at a micro-CT. Z Medical Physics. 27 (1), 56-64 (2017).

33. Newton, J. et al. Commissioning a small-field biological irradiator using point, 2D, and 3D dosimetry techniques. Medical Physics. 38 (12), 6754-6762 (2011).

34. Wong, J. et al. High-resolution, small animal radiation research platform with $\mathrm{x}$-ray tomographic guidance capabilities. International Journal of Radiation Oncology • Biology • Physics. 71 (5), 1591-1599 (2008).

35. Ghita, M. et al. Small field dosimetry for the small animal radiotherapy research platform (SARRP). Radiation Oncology. 12 (1), 204 (2017).

36. Biglin, E. R. et al. Preclinical dosimetry: exploring the use of small animal phantoms. Radiation Oncology. 14 (1), 134 (2019).

37. Munoz Arango, E. T., Peixoto, J. G., de Almeida, C. E. Small field dosimetry with a high-resolution 3D scanning water phantom system for the small animal radiation research platform SARRP: a geometrical and quantitative study. Physics in Medicine and Biology. (2019).

38. Murrell, D. H. et al. Evaluating Changes to Blood-Brain Barrier Integrity in Brain Metastasis over Time and after Radiation Treatment. Translational Oncology. 9 (3), 219-227 (2016). 